\title{
Anabases
}

ANABASES Traditions et réceptions de l'Antiquité

29 | 2019

Varia

\section{«La réception du théâtre antique dans les travaux savants de l'Europe de la Première modernité "}

\section{Malika Bastin-Hammou et Pascale Paré-Rey}

\section{(2) OpenEdition}

1 Journals

Édition électronique

URL : https://journals.openedition.org/anabases/8623

DOI : 10.4000/anabases.8623

ISSN : 2256-9421

\section{Éditeur}

E.R.A.S.M.E.

\section{Édition imprimée}

Date de publication : 14 avril 2019

Pagination : 89-91

ISSN : 1774-4296

\section{Référence électronique}

Malika Bastin-Hammou et Pascale Paré-Rey, « «La réception du théâtre antique dans les travaux savants de l'Europe de la Première modernité » », Anabases [En ligne], 29 | 2019, mis en ligne le 14 avril 2021, consulté le 06 novembre 2021. URL : http://journals.openedition.org/anabases/8623 ; DOI : https://doi.org/10.4000/anabases.8623 

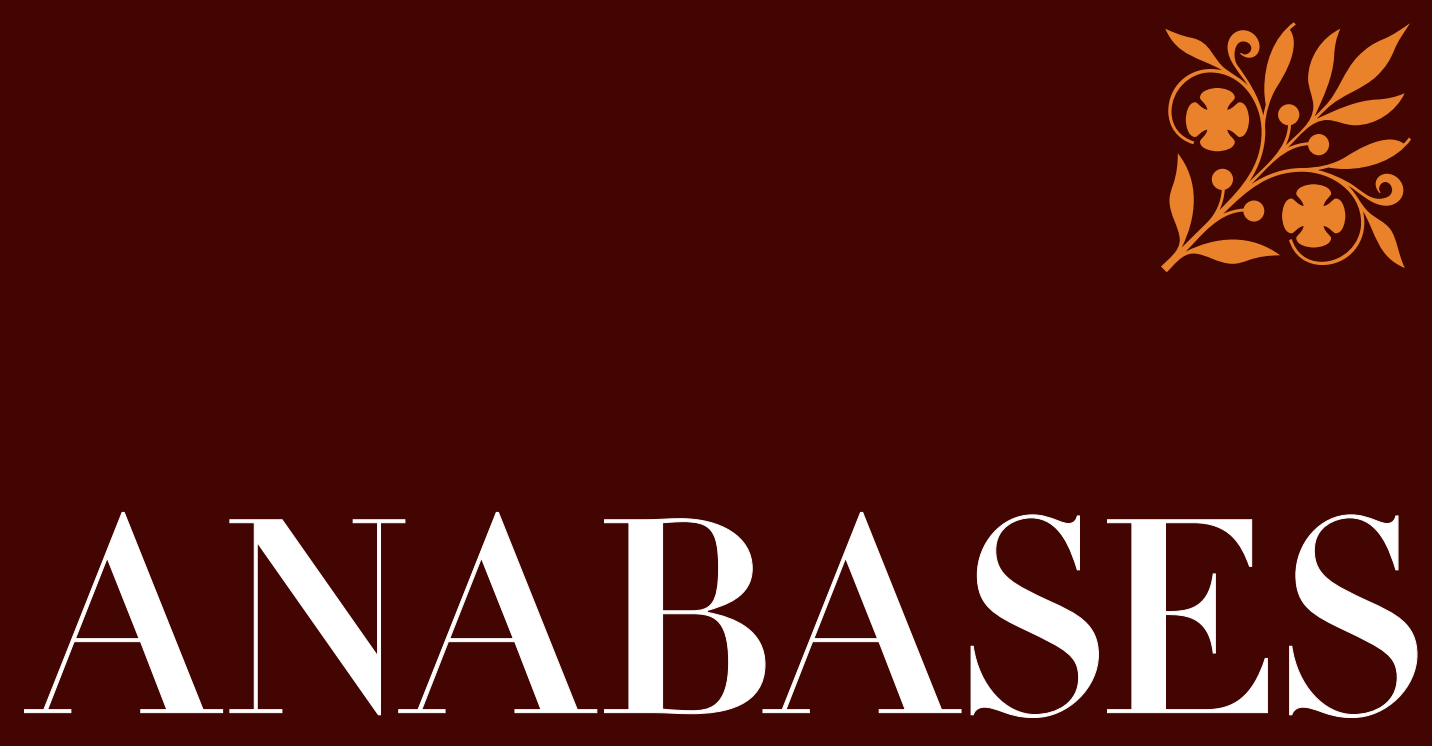

Traditions et Réceptions de l'Antiquité

\section{No29 \\ 2019}

Juliette Ernst Sculptures onctueuses de Meekyoung Shin Théâtre antique et travaux savants dans la Première modernité L'Antiquité dans la peinture (17911880) Réception d'Ovide Pierre Grimal 
ANABASES

Traditions et Réceptions de l'Antiquité

Revue de l'équipe de recherche E.R.A.S.M.E.

Université Toulouse-Jean Jaurès (UT2J)

Anabases dispose d'un Comité de lecture international. Chaque article envoyé à la rédaction est soumis, une fois anonymisé, à l'expertise de deux spécialistes qui rendent un rapport écrit. Les deux rapports anonymisés sont transmis à l'auteur qui tient compte des observations en vue de la publication.

\section{Comité SCIEnTIFIQUe}

Germaine Aujac (université Toulouse-Jean Jaurès : histoire de la géographie et des sciences antiques)

Florence Bouchet (université Toulouse-Jean Jaurès : littérature médiévale)

Hinnerk BruHns (CNRS : histoire économique et sociale ancienne et contemporaine)

Paulo Butti de Lima (université de Bari : historiographie et réception de l'Antiquité)

Luciano CANFora (université de Bari : littérature et histoire anciennes, historiographie)

Giovanna Ceserani (Stanford University : histoire intellectuelle et historiographie de la tradition classique)

Temístocles Cezar (université de Porto Alegre : historiographie moderne)

Serafina Сиомо (University of London, Birkbeck College : histoire des mathématiques et des sciences)

Paul Demont (université de Paris Sorbonne : philologie grecque et héritage classique)

Marie-Laurence Desclos (université de Grenoble II : philosophie de l'Antiquité)

Olivier Devillers (université de Bordeaux 3 - Michel-de-Montaigne : littérature et historiographie latines)

Andrea Giardina (Istituto italiano di scienze umane : histoire du monde romain et de ses réceptions)

Ève Gran-Aymerich (aibl : histoire de l'archéologie et des transferts culturels)

François HaRTog (EHEss : historiographie ancienne et moderne)

Geneviève Hoffmann (université de Picardie : histoire des mondes grecs)

Christian JACOB (CNRS/EHEss : histoire comparée et épistémologie des savoirs)

Suzanne Marchand (Louisiana State University : histoire du classicisme et de l'orientalisme)

Wilfried Nippel (Humboldt Universität Berlin : histoire et historiographie de l'Antiquité)

Sylvie Pittia (université de Paris I-Panthéon Sorbonne : histoire et historiographie du monde romain)

Stéphane Ratri (université de Franche-Comté - Besançon : philologie et héritage latin)

Comité de RÉdaction

Jacques Alexandropoulos, Marielle de Béchillon, Corinne Bonnet, Laurent Bricault, Clément Bur,

Philippe Foro, Adeline Grand-Clément, Anne-Hélène Klinger-Dollé, Véronique Krings,

Thibaud Lanfranchi, Claudine Leduc, Pascal Payen, Grégory Reimond, Catherine Valenti

Éditeur RESPonsable

Pascal PAYen

Université Toulouse-Jean Jaurès (UT2J)

SECRÉTARIAT DE RÉDACTION

Anthony Andurand / Clément Bertau-Courbières / Corinne Bonnet / Clément Bur /

Adeline Grand-Clément / Anne-Hélène Kuinger-Dollé / Véronique Krings /

Catherine Valenti (université Toulouse-Jean Jaurès) / Noémie VillacÈQue (université de Reims)

Sites Web

http://plh.univ-tlse2.fr

Revues.org : http://anabases.revues.org

Aвonnement et vente aU numéro

Éditions De Boccard - 4, rue de Lanneau - 75005 Paris

info@deboccard.com - www.deboccard.com

Tél. : 0033/(0)143260037 - Fax : 0033/(0)143548583 




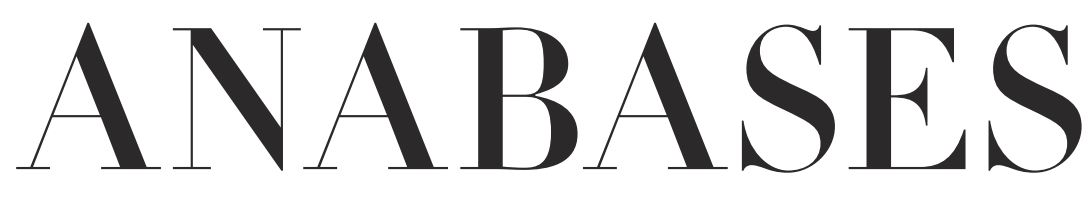

Traditions et Réceptions de l'Antiquité

$$
\begin{aligned}
& N \circ 29 \\
& 2019
\end{aligned}
$$

\section{E.R.A.S.M.E.}

Université Toulouse - Jean Jaurès 



\section{Sommaire}

$\mathrm{N}^{\circ} 29-2019$

\section{Historiographie et identités culturelles}

Ilse Hilbold

Les archives d'une bibliographe des sciences de l'Antiquité :

Juliette Ernst et la fabrique des relations internationales . . . . . . . . . I I3

Vivien LONGHI

La crise, une notion politique héritée des Grecs ? . . . . . . . . . . . 2I

Mireille Lacave-Allemand et Michel Lacave,

L’Antiquité dans la peinture en France, I79I-I880 :

une analyse quantitative à travers les Salons et les Prix de Rome . . . . . 37

Tiphaine Besnard

Du Weathering Project aux autoportraits en Venus :

Les sculptures onctueuses et savonneuses de Meekyoung Shin . . . . . 7 I

\section{Traditions du patrimoine antique}

Dossier dirigé par Pascale Paré-Rey et Malika Bastin-Hammou,

“La réception du théâtre antique dans les travaux savants de l’Europe

de la Première modernité »

Malika Bastin-Hammou et Pascale Paré-Rey

“ La réception du théâtre antique dans les travaux savants

de l'Europe de la Première modernité » . . . . . . . . . . . . . . . . 89

Kevin Bovier

Rétablir la métrique de Térence au $\mathrm{XvI}^{\mathrm{e}}$ siècle :

le cas du Iudicium de Glaréan (1540) . . . . . . . . . . . . . . . . . . . . . . 93 
Brice Denoyer

L'héritage de la métrique antique

dans l'alexandrin français au xvie siècle . . . . . . . . . . . . . IO7

Giovanna Di Martino

Vittorio Alfieri's tormented relationship with Aeschylus:

Agamennone between Tradition and Innovation . . . . . . . . . . . . . . . I2I

Marco Duranti

La condanna del prologo diegetico euripideo dagli scoli antichi

ai trattati del Cinquecento . . . . . . . . . . . . . . .

Rosario López Gregoris

L'influence de l' Arte nuevo de hacer comedias de Lope de Vega

dans l'usage des modèles classiques latins en Espagne

pendant le Siècle d'or et le Baroque » . . . . . . . . . . . . . . . . I49

Cressida Ryan

Sophoclean scholarship as a tool

to interpret eighteenth-century England . . . . . . . . . . . . . . г 6 I

Záviš ŠumaN

Axiologie critique de La Mesnardière . . . . . . . . . . . . . . . . . . . . I79

\section{Archéologie des savoirs}

Dossier dirigé par Cristina Noacco

“2000 ans déjà... Aspects de la réception d'Ovide » . . . . . . . . . . . I93

\section{La réception d'Ovide au Moyen Âge}

Jean-Marie Fritz et Cristina NoAcco

Lire Ovide au xiI ${ }^{\mathrm{e}}$ siècle : Arnoul d'Orléans

commentateur des Métamorphoses . . . . . . . . . . . . . . . $\quad{ }_{195}$

Franck Coulson

Le mythe de Pythagore dans le commentaire

“Vulgate » des Métamorphoses . . . . . . . . . . . . . . . . . . . . . . 2I

Marylène Possamaï

Comment éditer l'Ovide moralisé :

le problème de la mise en page du manuscrit Rouen Bm O.4 . . . . . . . 225

Anneliese Pollock Renck

Les Hérö̈des à la fin du Moyen Âge : pour une définition élargie de l'acte traducteur . . . . . . . . . . . . . . . . . . 239 
II. La réception d'Ovide à l'époque moderne

Fátima Díez Platas et Patricia Meilán Jácome

Le poète dans son œuvre. Ovide dans les images des Fasti

et des Tristia entre les Xv et $\mathrm{xvI}^{\mathrm{e}}$ siècles . . . . . . . . . . . . . . . . . 255

Ana Paula Rebelo Correia

Les représentations des Métamorphoses d'Ovide

dans les azulejos portugais. Influence des modèles gravés français . . . . 269

Sarah ReY

Figures d'Orphée au cinéma . . . . . . . . . . . . . . . . . . . 277

\section{Actualités et débats}

Marine LE BAIL

La modernité littéraire serait-elle affaire d'Antiquité(s) ?

Euvres \& Critiques: La contribution de l'archéologie à la genèse

de la littérature moderne, XLII, I, René Sternke dir., 20I7, 338 p. . . . . . . 2 29I

\section{Lire, relire la bibliothèque des sciences de l'Antiquité}

Éric Morvillez

“Les Horti Tauriani de Pierre Grimal

ou les prémices des Jardins romains » . . . . . . . . . . . . . . . . 30I

Pierre Grimal

“Les Horti Tauriani. Étude topographique sur la région

de la Porte Majeur ",MEFRA, tome 53, rg36. p. 25o-286 . . . . . . . . . . . 3 3i3

\section{L'atelier de l'histoire : chantiers historiographiques}

L'Antiquité au musée (coordonné par Adeline Grand-Clément) (6)

Aurélie Rodes, Catherine Valenti

Les Gaulois au musée . . . . . . . . . . . . . . . . . . . .

355

L'Atelier des doctorants (coordonné par Adeline Grand-Clément) (16)

Andrea Avalli

La question étrusque dans l'Italie fasciste $\ldots \ldots \ldots$. . . . . . . 360 
Droit et réception de l'Antiquité

(coordonné par Marielle de Béchillon et Hélène Ménard) (6)

Entre Clio et Thémis. Entretien avec Dario Mantovani, réalisé par

Hélène Ménard (Maître de Conférences d'Histoire romaine, à l'Université

Paul Valéry - Montpellier III), le 22 juin 20I8, à l'occasion de la parution

aux Belles Lettres du livre Les juristes écrivains de la Rome antique.

Les ouvres des juristes comme littérature (juin 20ı8) et de la création

de la chaire “ Droit, culture et société de la Rome antique »

au Collège de France $\left(\mathrm{I}^{\mathrm{er}}\right.$ novembre $\left.20 \mathrm{I} 8\right) \ldots$. . . . . . . . . . . . .

\section{Comptes rendus}

Philippe Borgeaud et Sara Petrella

Le singe de l'autre.

Du sauvage américain à l'histoire comparée des religions (A. Guedon) . . $\quad 37 \mathrm{I}$

Roberta Casagrande-Kim, Samuel Thrope et Raquel Ukeles (éd.)

Romance and reason. Islamic transformations of the classical past

(Cl. Bertau-Courbières) . . . . . . . . . . . . . . . . . . . . .

Hinnerk BruHns

Max Webers historische Sozialökonomie.

L'économie de Max Weber entre histoire et sociologie (Th. Lanfranchi) . . 374

Andrea Cozzo

Riso e sorriso, e altre saggi sulla nonviolenza nella Grecia antica,

(Fr. Pr. Barone) . . . . . . . . . . . . . . . . . . . 377

Franz Cumont

Manichéisme (St. Ratti) . . . . . . . . . . . . . . . 378

Emmanuelle HÉnin et Valérie NAAs (dir.)

Le mythe de l'art antique (Cl. Evrard) . . . . . . . . . . . . . . . . . 380

Jacques Jouanna, Henri Lavagne, Alain Pasquier,

Véronique SchiLtz et Michel Zink (éd.)

Au-delà du Savoir : Les Reinach et le Monde des Arts (G. Hoffmann) . . . .

382

Mario Liverani

Imagining Babylon: The Modern Story of an Ancient City (C.Bonnet) . . . 386

Françoise-Hélène Massa-Pairault, Claude Pouzadoux (Dir.)

Géants et Gigantomachie entre Orient et Occident (C.Giovénal) . . . . . . .

Scott McGill, Joseph Pucci (éd.)

Classics renewed. Reception and Innovation in the Latin Poetry

ofLate Antiquity (S. Clément-Tarantino) . . . . . . . . . . . . . . . . . . . 
Maxwell T. PAule

Canidia, Rome's First Witch (C. Landrea) . . . . . . . . . . . . . . . . . . . 39г

Jessica Priestley, Vasiliki Zali (éd.)

Brill's Companion to the Reception of Herodotus in Antiquity

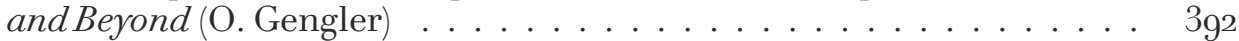

Salvatore QuAsimodo

La Lyre grecque $(\mathrm{M}$. Bianco) . . . . . . . . . . . . . . . . . . 395

Brett M. Rogers, Benjamin Eldon Stevens (éd.)

Classical Traditions in Modern Fantasy (M. Scapin) . . . . . . . . . . . . . 397

Maria Teresa Schettino et Céline UrLacher-Becht (dir.)

Ipse dixit. L'autorité intellectuelle des Anciens : affirmation,

appropriations, détournements (C. Psilakis) . . . . . . . . . . . . . 398

Guy G. Stroumsa

Religions d'Abraham : histoires croisées (D. Lorin) . . . . . . . . . . . . 400

Jean Yvonneau (éd.)

La Muse au long couteau. Critias, de la création littéraire

au terrorisme d'État (G. Hoffmann) . . . . . . . . . . . . . . 405

Résumés . . . . . . . . . . . . . . . . . . . . . 409

Index .............................. 423 



\section{Traditions}

\section{du patrimoine antique}

\section{Dossier dirigé par Pascale Paré-Rey et Malika Bastin-Hammou,}

“ La réception du théâtre antique dans les travaux savants de l'Europe de la Première modernité » 

Anabases 29 (2019), p. 89-91.

\title{
"La réception du théâtre antique dans les travaux savants de l'Europe de la Première modernité »
}

\author{
Malika Bastin-Hammou \\ et Pascale ParÉ-Rey
}

\footnotetext{
omment le théâtre antique a-t-il été lu, traduit, commenté, dans l'Europe de la Première modernité ? Le panel qui s'est tenu au sein de la Celtic Conference in Classics à Montréal en juillet 2017 s'inscrit dans l'intérêt
} actuel porté aux textes humanistes et classiques qui sont des vecteurs essentiels de sa redécouverte et de sa transmission ${ }^{2}$. L'angle choisi pour aborder cette réception était celui des travaux savants et plus précisément les textes liminaires - qu'ils soient extérieurs aux textes dramatiques eux-mêmes (épîtres dédicatoires) ou non (prologues) -, les traités développés (poétiques) ou les commentaires partiels (notes, scholies). Ces textes ont jusqu'alors été négligés ou sous-estimés, mais sont pourtant porteurs d'enseignements multiples par le regard critique qu'ils portent sur les pièces antiques. Ce regard critique, fait de réflexion et de jugement, a construit une vision qui n'est bien sûr pas uniforme mais a varié au cours de la période délimitée, selon les traditions nationales et au gré des conceptions de leurs auteurs, en fonction aussi des poètes et des pièces considérés.

En englobant la réception de tout le théâtre antique, grec et latin, tragique et comique, il s'agissait d'envisager comment et dans quelle mesure ces travaux ont été le lieu de réflexions soucieuses de comprendre et faire comprendre ce théâtre, mais aussi de saisir en quoi ils reflètent, orientent ou passent à côté des débats sur les théâtres vernaculaires contemporains. L'établissement du texte, la traduction le cas échéant, l'analyse, l'explication, la critique, l'indexation des pièces d'Eschyle,

1 voir https://www.celticconferenceclassics.com/reception-of-ancient-drama 
Sophocle, Euripide, Aristophane, ainsi que de Plaute, Térence et Sénèque, sont autant de tâches dont les érudits se saisissent, mus par des objectifs divers.

Les bornes choisies ont permis d'explorer des travaux savants de la fin du xve au $\mathrm{XVIII}^{\mathrm{e}}$ siècle, dans différents pays européens - Italie, France, Allemagne, Espagne, Angleterre, Pays-Bas - où le latin était la langue de la communication érudite ; plusieurs contributions portent ainsi sur des textes rédigés en latin. Ces limites entendaient surtout mettre en lumière la richesse de la production intellectuelle d'alors et orienter vers les pistes de recherche suivantes, soumises à la réflexion des contributeurs.

Ces derniers étaient ainsi invités à s'interroger sur des problématiques de langue : quels rapports ces travaux entretiennent-ils avec les langues anciennes? Sont-ils rédigés en latin ou en vernaculaire, et pourquoi ? A-t-on affaire à des traductions poétiques, littérales, ad verbum? Quel sort est réservé à la métrique ? Il nous a paru pertinent, par ailleurs, de replacer ces travaux dans leurs contextes historiques et politiques: quels intérêts, quels enjeux, voire quels risques comportent les démarches d'éditer, d'étudier, de jouer le théâtre antique dans une Europe pacifiée ou déchirée par les guerres de religion, les querelles de frontières ? Une autre piste de réflexion était celle du lien entre théâtre et spectacle : l'horizon de la scène est-il présent dans les textes étudiés? Comment jouer le théâtre grec, le théâtre latin? Quelles adaptations de la scène sont préconisées? Y a-t-il des textes injouables, et pourquoi ? Nous voulions réfléchir au rôle des travaux savants : quelles répercussions connaissent ces travaux savants? Quel impact ont-ils les uns sur les autres et sur les réceptions ultérieures du théâtre antique? Quels nouveaux concepts élaborent-ils? La question du rapport entre textes et lecteurs était également possible : ces textes sont-ils lus par les théoriciens du théâtre? Le public lettré ? Sont-ils le fait de professeurs? Sont-ils conçus en lien avec l'enseignement des langues anciennes? Du théâtre? Quelle pédagogie du théâtre donnent-ils à lire? On pouvait aussi considérer les éditeurs, traducteurs et imprimeurs : qui s'intéresse aux textes dramatiques de l'Antiquité ? Quelles sont les figures marquantes? Quels sont leurs liens avec le monde du théâtre? Quel est leur mode d'apparition à côté des / dans les textes théâtraux ? Enfin un horizon des études était celui de l'histoire du livre : comment relier la floraison de la littérature savante à l'histoire du livre? Quelles évolutions matérielles contraignent, expliquent, telle posture des commentateurs?

Tous ces axes n'ont bien sûr pas été abordés de façon égale et le champ de recherche reste encore largement ouvert. S'est dégagé un intérêt pour les questions techniques comme la place et le rôle de la métrique, dans les interventions de Kévin Bovier et de Brice Denoyer. Le premier, à partir du commentaire aux comédies de Térence de Heinrich Glaréan (1540), étudie les méthodes visant à corriger le texte et à en rétablir les mètres pour situer cette exégèse au sein du débat humaniste sur la métrique du comique latin. Le second se demande pourquoi c'est l'alexandrin 
qui s'est imposé au xvi ${ }^{\mathrm{e}}$ siècle comme le vers du dialogue tragique par excellence ; or une partie de la réponse est à chercher dans le lien entre la traduction des tragédies antiques et l'élaboration des tragédies en vernaculaire par les poètes français. Les auteurs ont été attentifs au contexte social et culturel dans lequel la lecture des classiques s'effectue et, en retour, à l'impact de celle-ci sur la tradition ultérieure. Rosario López Gregoris montre ainsi comment la théorie de Lope de Vega sur “l'art nouveau de faire des comédies » a contribué à éclipser les comiques latins sur la scène espagnole du $\mathrm{xvI}^{\mathrm{e}}$ siècle. C'est dans le contexte politique, culturel et pédagogique de l'Angleterre du xvIII $^{\mathrm{e}}$ siècle. que Cressida Ryan quant à elle situe les études sur Sophocle (éditions, apparat critique, préface, dictionnaire) en mettant en lumière les idées modernes qui s'en dégagent. De manière similaire à l'impact de l'art poétique de Lope de Vega, la Poétique de la Mesnardière, pour Záviš Šuman, a influencé la réception des tragiques grecs et latins dans la France du XvII ${ }^{\mathrm{e}}$ siècle. Enfin, on n'a pas manqué de souligner les positions franchement critiques ou du moins ambiguës des Modernes sur les Anciens : Marco Duranti reconstruit la condamnation des prologues diégétiques euripidéens depuis les commentateurs anciens eux-mêmes jusqu'aux traités du Cinquecento, tandis que Giovanna Di Martino présente la relation tourmentée d'Alfieri à Eschyle, entre appropriation par fidélité au modèle et distanciation par imprégnation de la modernité.

Ce volume présente tout d'abord les articles sur la comédie latine (Kévin Bovier et Rosario López Gregoris), puis sur la tragédie grecque (Marco Duranti, Giovanna Di Martino et Cressida Ryan) et enfin sur la poétique (Brice Denoyer et Záviš Šuman). Ces aperçus sont loin d'épuiser le sujet et l'on pourrait les prolonger en s'intéressant, par exemple, à d'autres figures de passeurs de textes, en cernant plus systématiquement les traditions nationales de réception du théâtre antique ou la façon dont les approches métriques et philologiques se sont élaborées et ont compté dans l'intelligence des textes dramatiques.

\author{
Malika Bastin-Hammou \\ Université Grenoble - Alpes \\ Litt\&Arts (UMR 5316) \\ malika.bastin@univ-grenoble-alpes.fr
}

\author{
Pascale Paré-Rey \\ Université de Lyon, \\ Jean Moulin - Lyon 3 \\ HiSoMA (UMR 5189) \\ pascale.rey@univ-lyon3.fr
}

